\title{
Elucidating the Role of Topological Constraint on the Structure of Overstretched DNA Using Fluorescence Polarization Microscopy
}

\author{
Published as part of The Journal of Physical Chemistry virtual special issue "W. E. Moerner Festschrift". \\ Adam S. Backer,* Graeme A. King, Andreas S. Biebricher, Jack W. Shepherd, Agnes Noy, Mark C. Leake, \\ Iddo Heller, Gijs J. L. Wuite, and Erwin J. G. Peterman*
}

Cite This: J. Phys. Chem. B 2021, 125, 8351-8361

Read Online

ABSTRACT: The combination of DNA force spectroscopy and polarization microscopy of fluorescent DNA intercalator dyes can provide valuable insights into the structure of DNA under tension. These techniques have previously been used to characterize $S$ DNA-an elongated DNA conformation that forms when DNA overstretches at forces $\geq 65 \mathrm{pN}$. In this way, it was deduced that the base pairs of S-DNA are highly inclined, relative to those in relaxed (B-form) DNA. However, it is unclear whether and how topological constraints on the DNA may influence the base-pair inclinations under tension. Here, we apply polarization microscopy to investigate the impact of DNA pulling geometry, torsional constraint, and negative supercoiling on the orientations of intercalated dyes during overstretching. In contrast to earlier predictions, the pulling geometry (namely, whether the DNA molecule is stretched via opposite strands or the same strand) is found to have little influence. However, torsional constraint leads to a substantial reduction in intercalator tilting in overstretched DNA, particularly in AT-rich sequences. Surprisingly, the extent of intercalator tilting is similarly reduced when the DNA molecule is negatively supercoiled up to a critical supercoiling density (corresponding to $\sim 70 \%$ reduction in the linking number). We attribute these observations to the presence of P-DNA (an overwound DNA conformation). Our results suggest that intercalated DNA preferentially flanks regions of P-DNA rather than those of S-DNA and also substantiate previous suggestions that P-DNA forms predominantly in AT-rich sequences.

\section{INTRODUCTION}

Detailed knowledge of the elastic and mechanical properties of DNA is essential for obtaining a complete understanding of nucleic-acid processing in vivo. Under physiological conditions, and in the absence of force, double-stranded DNA exists in the so-called B-form, which exhibits a length of $0.34 \mathrm{~nm} / \mathrm{bp}$. However, when stretched to a force of $\sim 65 \mathrm{pN}$, B-DNA undergoes a structural transition known as overstretching. ${ }^{1,2}$ The overstretching transition (OST) is characterized by a $70 \%$ elongation of the DNA at nearly constant force, in which the double helix unwinds cooperatively, resulting in either basepair-melted DNA or an underwound, base-paired structure termed S-DNA. Whether S-DNA or melted DNA forms during overstretching depends on factors such as the base-pair sequence, ionic strength of the buffer, and the temperature. ${ }^{3-8}$

The case described above assumes that the DNA is torsionally unconstrained (UC) and thus that the molecule is topologically free to unwind under the applied tension. The OST changes markedly when the DNA molecule is torsionally constrained (TC) due to the fact that the overall linking number ( $L k$, defined as the sum of the twist and writhe in the molecule) must remain constant. Consequently, the OST in TC-DNA occurs at much higher forces $(\sim 110 \mathrm{pN})$ than that in UC-DNA. This is because the presence of underwound structures (such as S-DNA) must be compensated for by a corresponding overwinding in other sections of the molecule. ${ }^{9}$ It has been proposed that this local overwinding arises via the formation of a structure known as Pauling (P)-DNA, in which the phosphate backbones wrap around one another with a helicity of $\sim 2.5$ bases per turn. The bases in P-DNA are thought to be unpaired and point outward. ${ }^{10}$ Based on the measured helicity of S-DNA $(\sim 37.5 \mathrm{bp} /$ turn $)$, and the proposed structure of P-DNA, these two conformations are

Received: March 26, 2021

Revised: July 1, 2021

Published: July 26, 2021

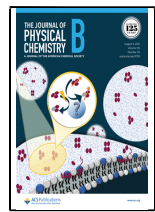



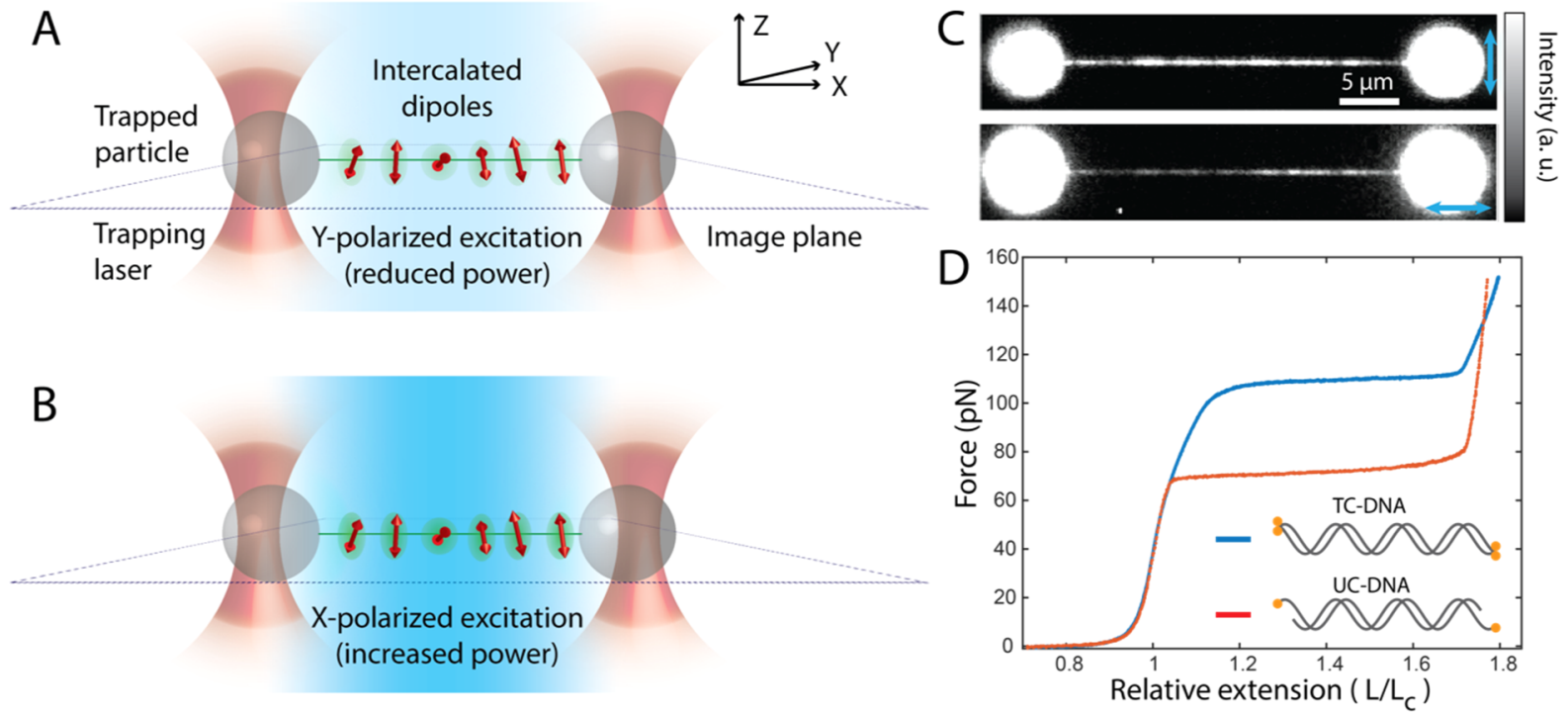

Figure 1. Overview of the experimental method. (A) Imaging protocol: an intercalated $\lambda$-DNA molecule is stretched to a desired extension along the $X$-axis using dual-trap optical tweezers (transition dipole moments of intercalated dyes are drawn as red bidirectional arrows). The construct is first imaged using a reduced-power $Y$-polarized excitation beam, efficiently exciting dyes oriented close to the plane perpendicular to the DNA axis (YZ-plane). (B) A second camera frame is recorded using $X$-polarized light at increased power. This polarization will inefficiently excite dyes oriented in the YZ-plane but provide suitable fluorescence signals for LD measurement. (C) Representative fluorescence image data recorded using a TC-DNA molecule using Y-polarized (top) and X-polarized (bottom) excitation. (D) Representative force-extension curves for TC-DNA (blue) and UC-DNA (red). These curves were collected on DNA in the absence of intercalated dyes. The inset illustrates the attachment geometries for TC-DNA and UC-DNA constructs.

estimated to coexist in a ratio of approximately 4:1 S-DNA:PDNA to ensure a constant $L k{ }^{9}$ This finding was supported by subsequent fluorescence microscopy studies of overstretched TC-DNA. ${ }^{11}$ Owing to the increased stability of GC base pairs relative to AT base pairs, it was suggested that P-DNA (which requires base-pair melting) primarily forms in AT-rich sequences of the DNA molecule, while S-DNA dominates in GC-rich sequences. ${ }^{11}$

When torsional stress is applied to TC-DNA, the overall $L k$ will either increase or decrease depending on the direction of the applied torque, resulting in DNA overwinding (positive supercoiling) or underwinding (negative supercoiling), respectively. The extent of supercoiling is defined by the relative change in $L k$, parameterized by the supercoiling density, $\sigma=$ $\left(L k-L k_{0}\right) / L k_{0}$. Here, $L k_{0}$ refers to the value of $L k$ in relaxed (B-form) DNA. In the case of negatively supercoiled DNA, less (overwound) P-DNA is required to offset (underwound) SDNA during overstretching. At a critical supercoiling density of $\sigma \sim-0.7$, no P-DNA is required during overstretching, and thus, at the end of the OST, the DNA will consist entirely of SDNA. 9

In order to characterize local changes in the structure of overstretched DNA, fluorescence imaging and force spectroscopy of intercalated DNA have proved highly informative. ${ }^{4,7,12-14}$ DNA intercalators are small planar dye molecules that bind to DNA by sliding between adjacent base pairs, which results in local unwinding and elongation of the double helix. ${ }^{15}$ Upon binding to DNA, intercalators align parallel to neighboring base pairs and undergo a fluorescence enhancement of two orders of magnitude or more. ${ }^{16-18}$ While intercalators strongly favor B-DNA binding, the absence of intercalator fluorescence has been used frequently to deduce the presence of non-B-form overstretched structures, such as $\mathrm{S}$ DNA. ${ }^{4,7,11-14}$
Polarized excitation and fluorescence imaging of intercalators can additionally reveal the orientations of the intercalated dyes' transition dipole moments relative to the axis of the DNA molecule. Previous studies have demonstrated that intercalated dyes orient perpendicular to the DNA axis at forces $<65 \mathrm{pN}$ and also for partially overstretched DNA (i.e., where less than $100 \%$ of the molecule has been overstretched). ${ }^{19-22}$ In these cases, B-DNA neighboring the intercalation site acts to align the intercalators in the same orientation as the base pairs in Bform DNA. Recently, ${ }^{23,24}$ we demonstrated that at extensions beyond the end of the OST, intercalated dyes tilt to an orientation of $\theta \sim 54^{\circ}$ relative to the DNA axis. At these extensions, most of the B-DNA has been converted to S-DNA due to the unwinding of the molecule. Although it is energetically unfavorable for intercalators to bind directly to S-DNA, our data suggested that dye molecules assume a tilted configuration when an intercalation site was neighbored by (unintercalated) S-DNA. Intercalator tilting was only observed at DNA extensions beyond the end of the OST, rather than during the OST (where S-DNA and B-DNA coexist) because it is energetically unfavorable for S-DNA (relative to B-DNA) to neighbor intercalated DNA. ${ }^{14}$ Taken together, these measurements led us to conclude that S-DNA exhibits highly inclined base pairs relative to $\mathrm{B}-\mathrm{DNA}^{24}$ and thus induces the tilting of flanking intercalated DNA. While this work provided valuable insights into the structure of S-DNA, these experiments considered only a single stretching geometry in which the tension was applied via opposing $3^{\prime}$ ends. However, it has been suggested that different base-paired overstretched structures may form depending upon whether tension is applied on the same or opposite DNA strands. ${ }^{25-30}$ Moreover, the influence of torsional constraint and supercoiling (and thus the effect of P-DNA) on S-DNA-induced intercalator tilting is unknown. 
Here, we apply intercalator polarization microscopy to explore the influence of pulling geometry, torsional constraint, and negative supercoiling on the structure of overstretched DNA. We show that intercalator tilting beyond the OST in UC-DNA is independent of whether the construct is pulled via the $3^{\prime}$ ends of opposite strands or the $5^{\prime}$ and $3^{\prime}$ ends of the same strand. This indicates that the tilted orientation of intercalated dyes is caused by the inclined base pairs within the $S$-DNA conformation and is not a consequence of a specific pulling geometry. We next demonstrate that at forces beyond the OST, intercalated dyes tilt significantly less in TC-DNA than in UC-DNA. Moreover, the extent of intercalator tilting in TC-DNA displays a strong sequence dependence, whereby dyes are tilted more strongly in GC-rich sequences compared with those in AT-rich DNA regions. This contrasts with the case of UC-DNA. Finally, we conducted similar fluorescence polarization measurements on negatively supercoiled TC-DNA using the recently reported technique of optical DNA supercoiling (ODS). ${ }^{31}$ These experiments reveal that over a narrow range of negative supercoiling densities $(-0.6<\sigma<$ $-0.7)$, the intercalated dye orientations beyond the OST exhibit a sudden transition from a sequence-dependent, slightly tilted state to a sequence-independent, highly tilted state. From these results, we draw two main conclusions: first, we confirm that P-DNA forms predominantly within AT-rich sections of overstretched TC-DNA. Second, we argue that it is more favorable for P-DNA, rather than S-DNA, to flank intercalator binding sites.

\section{METHODS}

2.1. Polarization Microscopy. Figure $1 \mathrm{~A}, \mathrm{~B}$ provides an overview of the experimental technique. A setup combining dual-trap optical tweezers and fluorescence microscopy was used to manipulate and extend single DNA molecules, tethered between two optically trapped beads. ${ }^{32}$ As described in refs 23 and 24, polarized laser illumination was used for fluorescence excitation. However, in a departure from our previous work, fluorescence emission was not resolved into its $X$-/Y-polarized components (see our rationale behind this change in the paragraph below). As in our previous experiments, ${ }^{23,24}$ we used bacteriophage $\lambda$ DNA ( $\lambda$-DNA), which has a contour length of $\sim 16.5 \mu \mathrm{m}$. We used the bis-intercalator YOYO-1 to label the DNA. A collimated $488 \mathrm{~nm}$ laser beam was used for widefield epifluorescence excitation. Laser intensities of $\sim 1-5 \mathrm{~W} / \mathrm{cm}^{2}$ were used for imaging experiments (estimated by measuring transmitted laser power by placing a power meter above the microscope objective and dividing by the size of the illuminated area at the sample). The polarization of the excitation laser was controlled using an EOM (model 350-80, Conoptics) placed in the beam path. By synchronizing the input voltage to the EOM with the camera exposure TTL signal, the excitation laser was rapidly toggled between $X$ - and $Y$-polarization during the read-out period after each successive camera frame was recorded. Additionally, the intensity of the polarized excitation laser was controlled using a liquid crystal beam attenuator (ThorLabs LCC 1620) placed before the EOM. This device was modulated after each camera exposure to compensate for polarization-dependent losses incurred as the beam propagated through the excitation pathway and achieve different intensities for $X$-/Y-polarized excitation (Figure 1A,B). The degree of polarization of the collimated excitation beam was measured to be $>98 \%$, and within $\sim 2^{\circ}$ of the $X$ - and $Y$-axes of the experimental reference frame, using a polarimeter (ThorLabs PAX 1000VIS) placed above the microscope objective. Raw fluorescence data (Figure 1C) were acquired using an Andor EMCCD (iXON 897) set to an electron multiplication gain of 100 using integration times of 1 s per camera frame for both $X$ - and $Y$-polarized excitation. A complete description of other aspects of the experimental apparatus may be found in the previous work. ${ }^{24}$

Here, we detail some key changes to our experimental approach that facilitated the study of TC-DNA and supercoiled DNA. A single nick in the DNA backbone will cause the entire construct to become unconstrained, irreversibly altering the force-extension behavior (Figure 1D) and limiting our ability to investigate conformations that require a fixed $L k$ before and during overstretching. Fluorescence imaging therefore had to be carried out under conditions that minimize the risk of photoinduced nicking upon the bleaching of intercalated dyes. Thus, it was paramount to limit the fluorescence excitation laser intensity and reduce the fluorescence excitation of intercalated DNA. Due to the need to conserve a dim fluorescence signal as much as possible, we departed from our previous approach by opting to not use a polarizing beam splitter in the imaging pathway of our microscope. In this imaging configuration, we no longer resolve the polarization of light emitted by intercalated dye molecules and instead rely on changes in total fluorescence intensity as a function of excitation polarization to gather information about the overall alignments of intercalated dyes. ${ }^{33-35}$ This technique uses changes in fluorescence emission to determine how efficiently intercalated dyes absorb $X$-versus $Y$-polarized excitation light and may be considered a linear dichroism measurement. The linear dichroism (LD) is calculated as

$$
\mathrm{LD}=\frac{{ }_{x}^{I-\varepsilon_{y} I}}{x I+\varepsilon_{y} I}
$$

In eq $1,{ }_{x, y} I$ are the background-subtracted fluorescence signals obtained using $X$-/Y-polarized excitation, respectively (to maintain consistency with our earlier work, we use prescripts to denote excitation polarization and omit postscripts since the emission polarization is not resolved). $\varepsilon$ is the correction factor that accounts for the difference in laser intensity used when alternately exciting with $X-/ Y$-polarized illumination, as well as the polarization-dependent transmission efficiency of the filters placed in the imaging pathway. The LD alone does not enable the absolute orientation of the intercalated dyes to be determined (as this would require the combined excitation/emission polarization-resolved measurements, which are difficult to perform without nicking the DNA, as discussed above). Nevertheless, the LD provided a highly streamlined means of inferring relative changes in dye orientation. Specifically, the LD can be used to detect when dyes are no longer aligned in the plane perpendicular to the DNA axis. Furthermore, the use of a single (unpolarized) imaging channel enabled LD values to be calculated on a pixelwise basis, without the need for image registration-allowing spatial variations in LD along the axis of the DNA to be readily identified.

An additional modification to our experimental technique involved using substantially reduced laser power (ratio of 1:5.42) when exciting with $Y$-polarized in contrast to $X$ polarized illumination (Figure 1A,B). The precise ratio of excitation power was determined by imaging a solution containing freely diffusing eGFP and measuring changes in 
fluorescence emission upon toggling the laser power/polarization. In conventional LD experiments, care is often taken to use near-equal excitation power for both excitation polarizations, leading to drastically different output intensities for well-ordered samples. In our case, however, increased output fluorescent signals went hand-in-hand with accelerated photonicking-it was therefore preferable to adjust the laser power such that the fluorescent signal was uniformly low (yet still detectable above the background) for both input polarizations and profit from a sufficiently low photo-nicking probability (allowing greater than $\sim 8 \mathrm{~s}$ of imaging time in some cases). Since the TC-DNA constructs primarily exhibited negative LD (absorption dipole moments located primarily in the plane perpendicular to the DNA axis), we reasoned that the laser power should be attenuated when using $Y$-polarized light to image an intercalated DNA molecule aligned along the $Y$-axis of the experimental system. This approach of using combined amplitude/polarization modulation had the additional benefit of amplifying small changes in the fluorescence signal resulting from $X$-polarized excitation (which would cause unnecessary photo-nicking or even the saturation of the camera sensor had identical power been used for the complementary $Y$-polarized excitation). The higher laser power used for $X$-polarized excitation was also necessary to obtain a sufficient fluorescent signal above the background to make an accurate estimate of LD. For all fluorescence imaging experiments, a $\sim 25-50 \mathrm{nM}$ concentration of the bis-intercalating dye YOYO-1 was used. YOYO-1 was selected because in our previous experiments, ${ }^{23,24}$ intercalator tilting was most readily observed under a wide range of experimental conditions due to the dye's slow unbinding kinetics at forces beyond the OST. A high-salt $(1 \mathrm{M} \mathrm{NaCl})$ buffer was always used for imaging. This imaging buffer ensured that overstretching the DNA constructs would favor S-DNA formation in contrast to base-pair melting. ${ }^{7,11}$

2.2. DNA Construct Design and Generation of Negatively Supercoiled DNA. All experiments were performed using a linearized $\lambda$-DNA construct $(\sim 48.5 \mathrm{~kb})$ which was end-labeled with biotin and tethered between streptavidin-coated microspheres $(\sim 4.5 \mu \mathrm{m}$, Spherotech). The topological state of these constructs varied depending on the biotin-labeling strategy. For opposite-stranded constructs, biotin labels were added to the $3^{\prime}$ ends of each strand using Klenow DNA polymerase exo to fill in the $5^{\prime}$ overhangs of $\lambda$ phage DNA with biotin-labeled nucleotides, as described previously. ${ }^{36}$ For the same-stranded UC-DNA constructs, biotins were positioned on the $3^{\prime}$ and $5^{\prime}$ ends of only one strand by ligating biotin-labeled oligonucleotides to each end of $\lambda$-phage DNA using the protocol detailed in ref 37 . TCDNA constructs were prepared by ligating a hairpin "end-cap" to each end of linearized $\lambda$-DNA, such that both ends of the molecule were closed. Each end-cap was labeled with four biotins near the tip and, in most cases, the end-closed construct was tethered to streptavidin-coated beads via at least two biotins on each end of the molecule. ${ }^{11}$ This prohibited the rotation of the DNA molecule with respect to the beads and thus rendered the molecule TC (note that the beads do not rotate in the optical traps).

The above-mentioned end-capped TC-DNA construct was also used to generate negatively supercoiled molecules using ODS. ${ }^{31}$ In brief, ODS exploits the mechanical properties of end-capped TC-DNA to induce a fixed reduction in the overall $L k$. This is achieved as follows: first, an end-capped TC-DNA molecule is stretched to forces $>150 \mathrm{pN}$. After a period of time (typically 5-20 s) at these high forces, one or more biotinstreptavidin linkages are transiently broken, leaving one end of the DNA molecule tethered to the beads via only a single biotin-streptavidin tether. During this time, the molecule is in a UC overstretched state. As a result, the $L k$ is reduced via swivelling of the DNA molecule around the single tether. When the broken biotin-streptavidin linkages re-form, the DNA molecule becomes TC again but with a lower $L k$ than that of B-form DNA. The reduced $L k$ is retained even when the tension is released, and therefore, the molecule is negatively supercoiled. The extent to which $L k$ is reduced depends on the duration that the biotin-streptavidin bonds are broken for. Through repeated stretch-release cycles, the $L k$ can be reduced by between $<5$ and $70 \%$. The supercoiling density can be quantitatively determined, based on the extension at which $70 \mathrm{pN}$ of force is applied to the DNA molecule, using a look-up table that relates the DNA extension at $70 \mathrm{pN}$ to the supercoiling density (see Figure $2 \mathrm{~B}$ of ref 31 ). In this way, the supercoiling density can be estimated with a precision of \pm 0.05 (however, factors such as the ionic strength of the imaging buffer and dye coverage can slightly bias these measurements). The maximum reduction in $L k$ is achieved when the entire DNA molecule is converted to S-DNA at high force through transient biotin-streptavidin ruptures of TCDNA.

2.3. Data Analysis. LD measurements were performed on sequences of camera exposures at least $2 \mathrm{~s}$ long (one complete frame for both $X$ and $Y$ polarizations) and for some sequences as long as $8 \mathrm{~s}$ (in which the four interleaved excitations using a given polarization were averaged together to yield an image with superior signal-to-noise). To perform LD measurement, the following procedure was used. A region of interest (ROI) was specified using a custom Matlab GUI by selecting the endpoints of a given DNA image (the locations where the DNA meets the trapped bead). The ROI was then defined as a line 5 camera pixels in diameter running between the two DNA endpoints. Two regions of identical size containing only fluorescent background were automatically defined by offsetting the ROI by \pm 5 camera pixels and smoothing out noise using a three-pixel Gaussian convolutional filter ( sigma $=1$ pixel). These two background regions were then averaged together to yield a pixel-wise map for background subtraction. Pixels containing background-subtracted signals less than a (heuristically chosen) threshold of $100 \mathrm{ADC}$ counts were excluded from further analysis. This process was performed for both images corresponding to the $X$ - $/ Y$-polarized signal using an identical ROI-permitting LD to be calculated on a pixelwise basis without the need for image registration using eq 1 . LD images were generated using a custom HSV colormap in which hue was used to denote $\mathrm{LD}$, and the value was linearly scaled according to the fluorescence intensity $\left({ }_{x} I+\varepsilon_{y} I\right)$. Regions of the image not containing the ROI were desaturated (made black-and-white) to avoid distraction. To plot LD as a function of DNA position, the "fractional DNA position" was defined as the position of a pixel relative to the endpoints of an ROI, with 0 corresponding to the leftmost point and 1 corresponding to the rightmost. Individual pixels were plotted as dots with respect to their fractional position. To aid the viewer, smoothed data were also plotted as a solid line using a Savitsky-Golay filter using default settings. ${ }^{38}$ In this analysis, pixels having a fractional DNA position less than 0.05 or greater than 0.95 were excluded since it was reasoned that the 
A

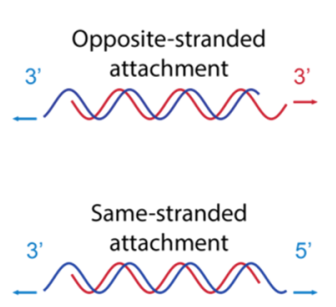

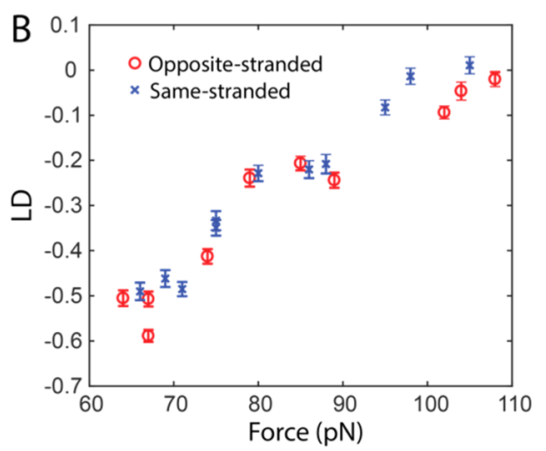

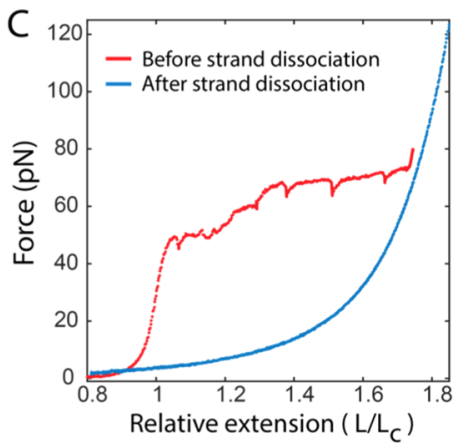

Figure 2. LD measurements of UC-DNA as a function of force for different pulling geometries. (A) Schematic of the DNA constructs used. An opposite-stranded attachment geometry was prepared, whereby a $\lambda$-DNA molecule was tethered to the optically trapped beads via the $3^{\prime}$-ends on opposite strands. The same-stranded attachment was prepared by tethering the DNA molecule to the beads via the $5^{\prime}$ and $3^{\prime}$ ends of only one strand. (B) LD as a function of the applied force for both opposite- and same-stranded attachment geometries (red circles and blue $x$ 's, respectively). (C) Verification procedure for ensuring the same-stranded attachment: after fluorescence imaging, the DNA molecule was moved to a low-salt buffer and overstretched to $L / L_{C}>1.7$, resulting in the peeling of the nontethered DNA strand from the tethered strand. The red forceextension trace shows the expected saw-tooth pattern during overstretching associated with the peeling process, while the blue trace shows the corresponding curve for single-stranded DNA obtained after retracting the end-to-end length.

LD would be corrupted by the strong background signal emanating from the trapped beads. To compute the averaged LD across a portion of a DNA molecule, the mean LD from multiple pixels was determined, and confidence bars were drawn as the standard deviation, divided by the square root of the number of pixels used to compute the average. A given DNA molecule image generally contained $\sim 1000$ camera pixels used for $\mathrm{LD}$ calculation. To compute averaged LD in alternately GC-rich or AT-rich regions of the DNA, ${ }^{39}$ pixels with a fractional DNA position between 0.05 and 0.55 were designated as AT-rich and those with a fractional position between 0.55 and 0.95 were designated as GC-rich. The orientation of the $\lambda$-DNA could be deduced by inspecting raw fluorescence images of TC-DNA obtained using $X$-polarized laser excitation and noting that one side of the $\lambda$-DNA $(\sim 45 \%$ of the entire construct) exhibited brighter emission (corresponding to the GC-rich region).

\section{RESULTS}

3.1. Same-Stranded Pulling Geometry Does Not Affect Intercalator Tilting. To assess the influence of attachment geometry (see Figure 2A) on the base-pair inclination in UC-DNA beyond the OST, we prepared $\lambda$ DNA constructs in which only one strand of the duplex DNA molecule was tethered to the beads by labeling the $3^{\prime}$ and $5^{\prime}$ ends of only one of the two complementary DNA strands with biotin (same-stranded pulling geometry). This contrasted with our earlier measurements, where the DNA molecule was labeled with biotin on the $3^{\prime}$ ends of opposing strands (opposite-stranded pulling geometry). ${ }^{23,24}$ We then stretched these constructs to beyond the OST in a high-salt buffer containing YOYO-1 and measured the $\mathrm{LD}$ as a function of the applied force (Figure 2B). To ensure that the same-stranded pulling geometry was maintained throughout the LD measurements, it was important that the photoinduced nicking of the tethered strand did not occur. Therefore, the DNA molecule was transferred to a low-salt $(15 \mathrm{mM} \mathrm{NaCl})$ buffer (in the absence of dye) after each fluorescence imaging experiment and then restretched. Under these conditions, strand peeling during overstretching is strongly favored over S-DNA formation, and thus for extensions beyond the OST $\left(L / L_{c}>\right.$ 1.7 ), the nontethered strand will peel away from the tethered strand, yielding a purely single-stranded DNA construct (Figure 2C). However, if a nick was present in the tethered strand, the molecule would break during strand peeling. LD measurements were only considered for molecules where no nick was detected in the tethered strand. For nick-free molecules, LD measurements were collected under identical imaging conditions as for DNA attached using the oppositestranded pulling geometry. To ensure that data sets were fully comparable, we recorded a new set of opposite-stranded stretching curves rather than using the data reported in ref 24 . Note that the nick-screening protocol described above for the same-stranded pulling geometry cannot be used for oppositestranded pulling. However, in our current and previous measurements, ${ }^{23,24}$ we have observed no indication that photo-nicking of a DNA molecule initially prepared for opposite-stranded pulling leads to a detectable change in fluorescence polarization or LD. Here, we observed very similar LD measurements for both pulling geometries, and in each case, we observe fluorescence depolarization at the end of the OST, as previously reported for the opposite-stranded pulling geometry. ${ }^{23,24}$ ANOVA analysis was performed by fitting a linear model to the plotted data, and the null hypothesis was confirmed ( $p$-value of 0.27$).{ }^{40}$ Thus, the observed LD values at extensions beyond the OST indicate a strong inclination of the DNA base pairs, independent of the specific attachment geometry.

3.2. Torsional Constraint Inhibits Intercalator Tilting. We next applied our combined fluorescence polarization and force spectroscopy approach to probe how the tilt angle in overstretched DNA is affected by the presence of torsional constraint. In these experiments, a TC-DNA molecule was generated by tethering an end-capped (i.e., topologically closed) $\lambda$-DNA molecule between two optically trapped beads, via at least two biotin moieties on each end, as described previously. ${ }^{11}$ The presence of torsional constraint was verified based on the measured force-extension curve. In contrast to UC-DNA, where the OST is characterized by a plateau at $\sim 65 \mathrm{pN}$, the OST in TC-DNA occurs at much higher forces, ranging from $\sim 110$ to $130 \mathrm{pN}$ (Figure 1D).

We first performed LD measurements on TC-DNA at extensions within the OST $\left(L / L_{c}=1.2-1.55\right)$, yielding LD measurements between -0.76 and -0.81 , consistent with 
A
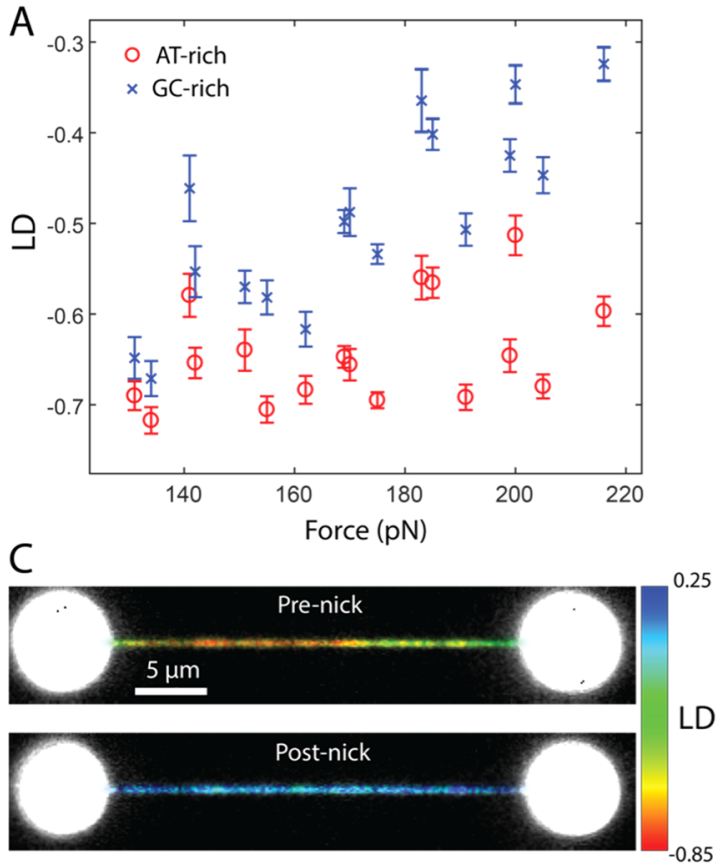

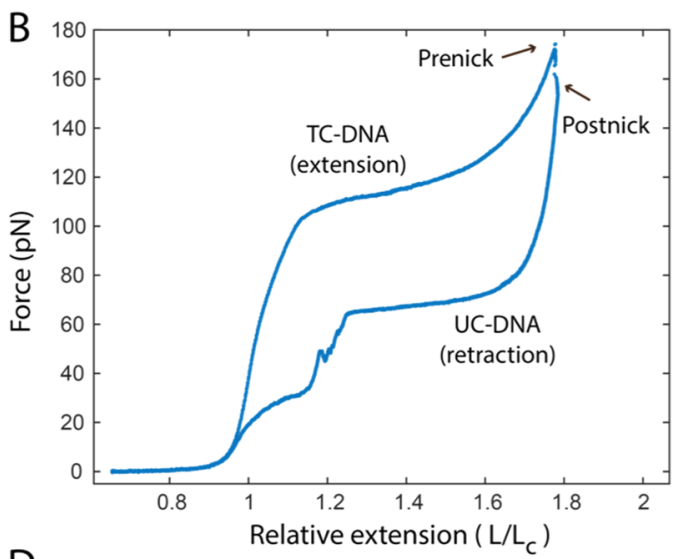

D
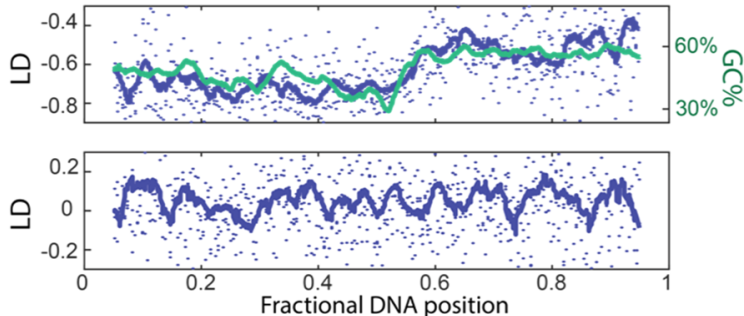

Figure 3. LD measurements for TC-DNA. (A) LD for TC-DNA as a function of the applied force associated with the AT-rich (red) and GC-rich (blue) regions of the $\lambda$-DNA construct. (B) Conversion of TC-DNA to UC-DNA via photo-nicking. The force-extension curve for intercalated TCDNA is shown prior to the occurrence of a nick. After waiting at $\sim L / L_{\mathrm{c}} \sim 1.75$ for a short period of time, a photo-induced nick occurs, resulting in a decrease in the force. The subsequent retraction curve is characteristic of UC-DNA containing a nick. Note that the force-extension behavior associated with intercalated DNA is slightly altered relative to that of bare DNA. ${ }^{12,13}$ (C) Fluorescence polarization images acquired immediately before and after photo-nicking at $\sim L / L_{\mathrm{c}} \sim 1.75$. (D) $\mathrm{LD}$ associated with the fluorescence images before (top) and after (bottom) photo-nicking occurred at $\sim L / L_{c} \sim 1.75$. Smoothed pixel-wise data are drawn as blue lines. To better illustrate the sequence dependence in the upper image, the rescaled locally smoothed percentage of GC base pairs as a function of the position in the DNA molecule is plotted in green.

intercalated dyes oriented nearly perpendicular to the DNA axis. We then investigated whether intercalator tilting could be detected in TC-DNA at extensions beyond the OST. To this end, we stretched TC-DNA to extensions ranging from $L / L_{c}=$ 1.7 to 1.84 (corresponding to the forces between 130 and 215 $\mathrm{pN}$ ). In contrast to UC-DNA, the LD values for TC-DNA beyond the OST exhibited a large magnitude and remained negative, even at extensions well beyond $L / L_{c}=1.7$. This indicates that the intercalated dye molecules retained orientations along (or close to) the plane perpendicular to the DNA axis even when the molecule was extended beyond the end of the OST. Nonetheless, when the DNA was stretched further, to extensions greater than $L / L_{c} \sim 1.75$ and forces $>130 \mathrm{pN}$ (see Figure 3A), the LD did eventually begin to decrease in magnitude (i.e., become less negative). However, these LD values were still significantly lower than those obtained from UC-DNA at a similar relative extension beyond the OST. For TC-DNA, the highest LD values were between -0.7 and -0.3 (depending upon the sequence-see below), with a typical precision of $\pm 0.03 \mathrm{LD}$ units (Figure 3A). This contrasts with the UC-DNA results (Figure 2B), where the maximum $L D$ reached values between -0.1 and 0.05 , with similar measurement precision.

A close inspection of the fluorescence data revealed that the LD values for TC-DNA exhibited reproducible patterns along the length of the DNA construct, in which one-half of the DNA molecule displayed a noticeably higher LD than the other half. This pattern correlated well with the known AT/ GC content of $\lambda$-DNA, which varies strongly between the two halves of the molecule (see the top panel, Figure 3D). In contrast, the LD in the case of UC-DNA varied over a much narrower range and showed no significant sequence dependence. To quantify the observed sequence dependence of the LD data in the case of TC-DNA, the transition point between low and high $\mathrm{LD}$ regions in each fluorescence image was determined by the eye, and the mean LD on either side of the transition point was calculated. These data are shown as a function of force in Figure 3A and reveal that AT-rich TCDNA retains a large magnitude, negative $L D$ of $\sim-0.6$ even at forces far beyond those encountered within the OST (up to $215 \mathrm{pN}$ ), while a more gradual decrease in the LD magnitude occurs as a function of force in the GC-rich portion of the molecule up to the values of $\sim-0.35$.

In order to confirm that the above-mentioned results were indeed a consequence of torsional constraint, the following control experiment was performed. Here, a single intercalated TC-DNA molecule was stretched to a relative extension of $L / L_{\mathrm{c}}=1.78$ (corresponding to a tension of $172 \mathrm{pN}$ ). This molecule was repeatedly imaged until a photoinduced nick was generated, and the construct promptly became UC, resulting in a rapid force drop of $\sim 20 \mathrm{pN}$ (Figure 3B). LD images collected immediately before and after formation of the photonick are shown in Figure 3C. Before nicking occurred, highly negative $\mathrm{LD}$ values were observed, and the magnitude of the LD was strongly correlated with the relative AT/GC content. However, from the fluorescence images taken immediately after nicking occurred, the LD increased in magnitude to roughly zero (very similar to the values obtained from UCDNA previously, Figure $2 \mathrm{~B}$ ) and no longer exhibited a strong correlation with AT-/GC-rich sequences. An additional 


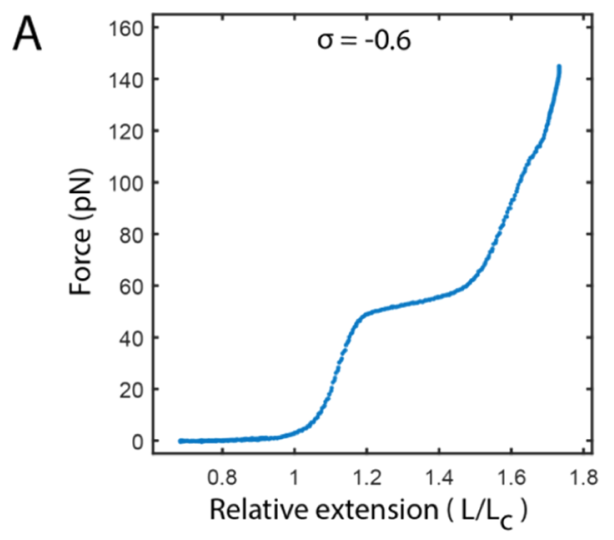

B

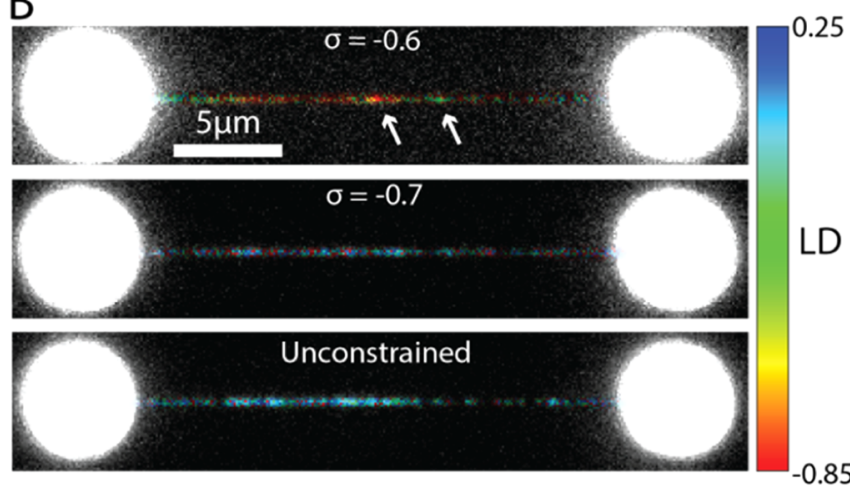

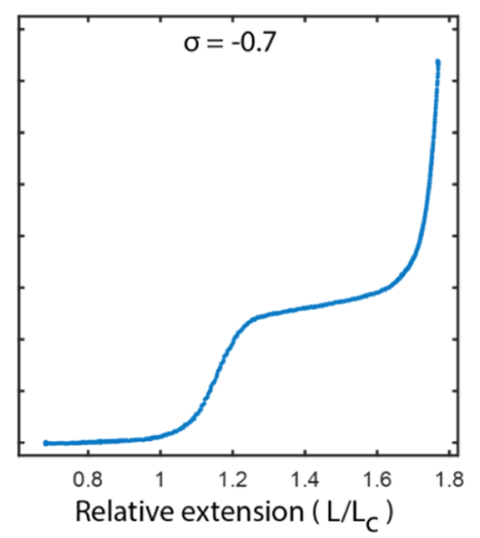

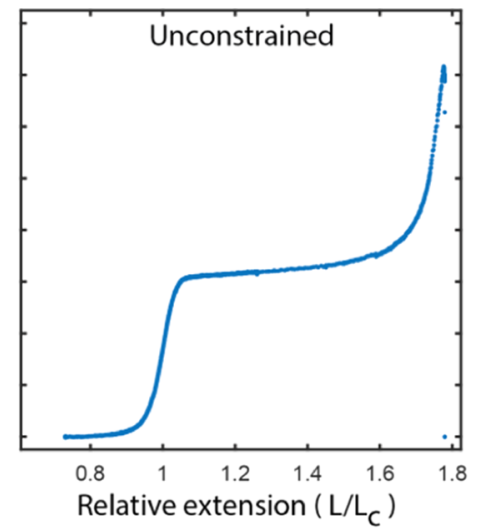

C

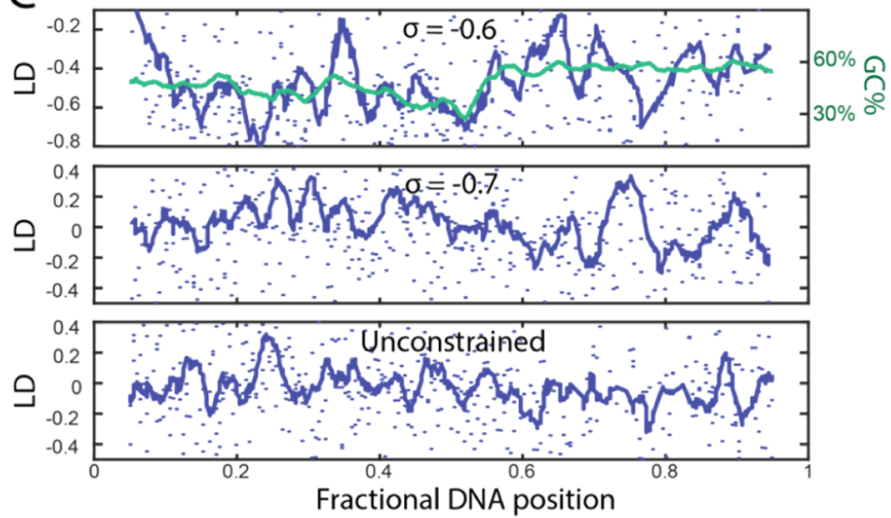

Figure 4. Comparison of LD beyond the OST as a function of negative supercoiling. (A) Sample force-extension curve for a molecule with (left) $\sigma$ $=-0.6$, (middle) $\sigma=-0.7$, and (right) after photo-nicking of a TC-DNA molecule when it was at $\sigma=-0.7$. Note that these curves were recorded sequentially for the same DNA molecule. (B) Fluorescence images showing the LD of a DNA molecule at (top) $\sigma=-0.6$, (middle) $\sigma=-0.7$, and (bottom) after photo-nicking, obtained sequentially from the same molecule. White arrows (top panel) indicate the regions of sequence-dependent LD. (C) LD values obtained from the DNA molecule for each case in B as a function of the fractional DNA position.

example of sequence-dependent LD is shown in Figure S2 in the Supporting Information. Note that in this additional example, the orientation of the construct is flipped (i.e., the $\mathrm{GC}$-rich portion is on the left side of the molecule).

3.3. Influence of Negative Supercoiling on Intercalator Tilting. We next investigated if, and to what degree, torsional stress influences the orientation of intercalators in TC-DNA. To this end, we generated underwound (negatively supercoiled) DNA, up to a maximum of $\sigma=-0.7$, using the recently developed technique of ODS. ${ }^{31}$ As the supercoiling density increases toward $\sigma=-0.7$, an increasingly small fraction of P-DNA is required to offset the formation of $S$ DNA during overstretching; ${ }^{9}$ beyond $\sigma=-0.7$, no P-DNA forms. ${ }^{9,31}$ This raises an intriguing question: how does the presence or absence of P-DNA in negatively supercoiled DNA alter the observed LD? To answer this, we measured the LD over a range of supercoiling densities from $\sigma=0$ to -0.7 at extensions beyond the OST (corresponding to forces of $\sim 150$ $\mathrm{pN}$ ). Surprisingly, no significant difference in intercalator tilting was observed up until at least $\sigma=-0.6$, even though only a small fraction of P-DNA remains at this high level of supercoiling ( $\sim 3 \%$ of the entire construct).

The force-extension curves for DNA with $\sigma=-0.6$ and -0.7 are shown in the left and middle plots of Figure 4A, respectively. The corresponding fluorescence polarization data are displayed in the upper two images of Figure 4B. At $\sigma=-0.6$, we obtained LD values between approximately -0.6 and -0.4 throughout the DNA molecule, largely consistent with the measurements obtained from nonsupercoiled TCDNA under identical conditions (Figure 3D). Similar to the case of nonsupercoiled TC-DNA, a notable sequence dependence of the LD values was observed for the LD associated with $\sigma=-0.6$. The most negative values of LD were obtained in the most AT-rich regions of the molecule (as shown in Figure 4B, upper panel, by the left white arrow at the fractional DNA position of approximately 0.55 ). The LD increases (becomes less negative) in GC-rich regions of the same construct (as indicated in Figure 4B, upper panel, by the right white arrow at the fractional DNA position of approximately 0.65). Our LD measurements at a supercoiling density of $\sigma=-0.6$ thus yield similar results to those obtained for nonsupercoiled TC-DNA and indicate that even a small fraction of P-DNA causes the intercalated dyes to assume orientations nearly perpendicular to the DNA axis, even at forces beyond the OST. An additional example, showing a DNA molecule with a supercoiling density of $\sigma=-0.25$, is shown in Figure S3. This DNA molecule also exhibits LD values similar to those obtained for TC-DNA.

When the supercoiling density was increased to $\sigma=-0.7$ (where no P-DNA was expected), a sudden and drastic change in the measured LD beyond the OST was observed. Here, the $\mathrm{LD}$ values were close to 0 throughout the molecule and the $\mathrm{LD}$ showed little or no sequence dependence, similar to the results observed for UC-DNA. This similarity was highlighted when the molecule with $\sigma=-0.7$ became (accidentally) nicked (due to photodamage). The occurrence of a nick was determined based on the change in force (yielding a force-extension curve 
consistent with that of UC-DNA, as shown in the right panel of Figure 4A). Upon switching from supercoiled TC-DNA (with $\sigma=-0.7)$ to UC-DNA, no significant change in LD beyond the OST was observed (see the lower two images in Figure $4 B, C)$. This suggests that in these cases, highly inclined DNA base pairs exist uniformly throughout the entire construct, in contrast to both nonsupercoiled TC-DNA and supercoiled TC-DNA with supercoiling densities of at least $\sigma=-0.6$. An additional example of a DNA molecule with a supercoiling density of $\sigma=-0.7$ is shown in Figure S4. This DNA molecule also exhibits $\mathrm{LD}$ values indicative of pronounced dipole tilting.

\section{DISCUSSION AND CONCLUSIONS}

In this study, we have used fluorescence polarization microscopy of DNA intercalators to characterize the structural features of overstretched DNA arising from pulling geometry, torsional constraint, and negative supercoiling. We first reveal that for intercalated UC-DNA, the LD values at extensions beyond the OST (e.g., at forces $>100 \mathrm{pN}$ ) are close to zero, independent of whether the molecule is overstretched via the same or opposite strands. This result demonstrates that the pronounced $\left(\theta \sim 54^{\circ}\right)$ intercalator tilting previously identified beyond the OST for UC-DNA does not depend on the specific pulling geometry but is most likely a direct consequence of the presence of S-DNA. This may, at first, seem surprising, given that several publications have predicted that the pulling geometry can alter the DNA structure under tension. ${ }^{29,30}$ Nevertheless, many of these predictions were based on molecular simulations that only considered very short dsDNA constructs $(<50 \mathrm{bp})$. Thus, our results could indicate that on much longer constructs $(\gg 10,000 \mathrm{bp})$, at least, any influence of the pulling geometry is no longer significant. We note that we cannot exclude the possibility that the pulling geometry might have an influence on DNA structural transitions during the OST; however, any effects would have to be sufficiently subtle that we cannot detect them with our approach.

Moreover, we have demonstrated here that intercalated TCDNA exhibits significantly lower (greater magnitude) LD values beyond the OST than UC-DNA. This indicates that the intercalated dyes remain oriented near the plane perpendicular to the DNA axis, even beyond the OST. We also established that at high forces $(>130 \mathrm{pN})$, the LD values for TC-DNA are sequence-dependent: although the dyes are largely perpendicular to the DNA axis, they are more tilted in GC-rich regions than in AT-rich regions. Furthermore, we explored the influence of negative supercoiling on intercalator tilting, revealing an intriguing observation: under conditions where no P-DNA is present during overstretching $(\sigma=-0.7)$, we observe essentially the same LD behavior as obtained for UCDNA, namely, intercalator tilting at the end of the OST, with no detectable sequence dependence. Strikingly, however, only a slight decrease in the supercoiling density (from $\sigma=-0.7$ to -0.6) was sufficient to restore the LD features observed for TC-DNA, that is, a greatly reduced intercalator tilting that is sequence-dependent.

To explain the observed LD measurements on TC-DNA, we first recapitulate one of the conclusions from our earlier work. ${ }^{24}$ There, it was noted that a significant depolarization of intercalator fluorescence was observed for UC-DNA only at the very end of the OST. That observation was explained by the assumption that (a) the observed depolarization (ascribed to intercalator tilting) is caused by the strongly inclined base pairs associated with the S-DNA conformation and (b) there is an energy penalty associated with S-DNA flanking intercalated B-DNA, as has been demonstrated previously. ${ }^{14}$ Hence, intercalated DNA is forced to neighbor S-DNA only when B-DNA has disappeared, that is, at extensions beyond the end of the OST, causing the intercalated dyes to assume tilted orientations.

In the case of TC-DNA, there are three relevant DNA conformations to be considered during the OST: B-DNA, SDNA, and P-DNA. While the existence of (overwound) PDNA is necessary to allow for the formation of (underwound) S-DNA, P-DNA is only present in a small fraction of the molecule. In the absence of supercoiling, the ratio of S-DNA:PDNA is roughly 4:1.,11 Since P-DNA is thought to exhibit a base-pair-melted structure, in which the bases are flipped outward, ${ }^{10}$ we assume that P-DNA will not influence the dye tilt angle at adjacent intercalation sites and that any neighboring intercalated dyes will have orientations perpendicular to the DNA substrate. This hypothesis is supported by the observation that beyond the OST, intercalators adopt a less tilted orientation in AT-rich (compared with GC-rich) regions of TC-DNA. This is consistent with previous suggestions that P-DNA is preferentially formed in AT-rich sequences. ${ }^{11}$

While the above-mentioned assumption that P-DNA will not cause neighboring intercalated dyes to tilt would qualitatively explain a relative decrease in LD (increase in magnitude), this assumption alone does not completely account for our observed results. If intercalated dyes were randomly distributed throughout an overstretched TC-DNA construct containing $20 \%$ P-DNA (perpendicular dyes) and 80\% S-DNA (tilted dyes), this would lead to an estimated LD of approximately -0.12 . This calculation is based on our previously developed mathematical model and experimentally measured tilt angles and "wobble" cones associated with intercalated UC-DNA extended within and beyond the OST (see the Supporting Information of ref 24 and refs 41-46). If we additionally assume that S-DNA must neighbor both sides of an intercalation site in order to cause significant dipole tilting, this would lead to a construct containing roughly $32 \%$ perpendicular dyes and $64 \%$ tilted dyes-yielding an estimated $\mathrm{LD}$ of only -0.21 . In contrast, our measured $\mathrm{LD}$ values are significantly lower than this: at the highest forces and extensions for which data were obtained, we recorded LDs of approximately -0.35 and -0.6 for GC-rich DNA and ATrich DNA, respectively.

We therefore propose that the most plausible explanation for the greatly reduced tilting measured in TC-DNA beyond the OST is that intercalated dyes are predominantly flanked by PDNA rather than by S-DNA. This hypothesis is further reinforced by the observed dependence of the LD on the supercoiling density. As the density of negative supercoiling is increased, the proportion of P-DNA in the molecules becomes increasingly small. However, even at $\sigma=-0.6$, where only a $\sim 3 \%$ total fraction of P-DNA is assumed to be present, ${ }^{31}$ we still observed a largely inhibited dye tilting beyond the OST. Only at a supercoiling density of $\sigma=-0.7$, that is, under conditions where P-DNA is expected to have completely disappeared, does the measured LD values approach the values obtained for UC-DNA. We propose that these observations can be explained by an assumption of a nonstochastic distribution of DNA states, in which intercalated DNA is strongly biased toward neighboring P-DNA rather than SDNA. This is consistent with the large energy penalty that was 
found previously for intercalated DNA neighboring S-DNA, ${ }^{14}$ whereas we expect no such penalty for P-DNA. This proposed model is summarized in Figure 5A,B.

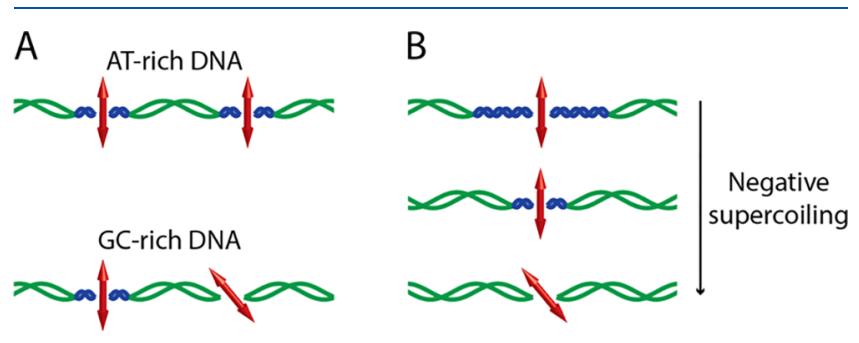

Figure 5. Proposed mechanisms by which P-DNA inhibits the tilting of intercalated dyes during overstretching. P-DNA is depicted in blue and S-DNA in green. Transition dipole moments associated with intercalated dyes are represented as red arrows. For simplicity, DNA bases are not drawn. (A) Due to the increased stability of GC relative to AT base pairing, P-DNA predominantly forms in AT-rich DNA. In AT-rich sections, there is sufficient P-DNA density such that intercalators are allowed to retain the energetically favored perpendicular orientation. Nevertheless, in GC-rich regions, S-DNA is intermittently "forced" to flank intercalated DNA, leading to sequence-dependent LD measurements and intercalated dipole tilting. (B) Negative supercoiling will reduce the total amount of P-DNA present in overstretched TC-DNA. However, due to the energetic penalty associated with S-DNA flanking intercalated DNA, any remaining P-DNA will preferentially flank intercalation sites. Once a construct approaches a sufficiently negative supercoiling density that P-DNA no longer forms during overstretching, the intercalated dipoles will be tilted (due to being flanked only by S-DNA) and the LD will approach the values recorded for UC-DNA.

We note that in all our experiments conducted using TCDNA, we utilized higher dye concentrations and lower laser illumination powers than those used in our previous experiments utilizing single YOYO-1 dye molecules. This was a concession made to reduce the probability of photo-nicking over imaging periods lasting on the order of $\sim 2-8 \mathrm{~s}$. However, under these conditions, we were unable to spatially resolve single intercalated dye molecules. As a result, our reported LD measurements were local ensemble averages over many dyes, and we were unable to detect whether individual intercalators assumed a discrete set of distinct (tilted/untilted) orientations, as was found to be the case in ref 24. Despite the increased dye concentration, the dye coverage is below saturation and, at the forces of interest, low enough that we do not expect significant influence on the overstretching characteristics of DNA. In this work, a YOYO-1 concentration of $\sim 25-50 \mathrm{nM}$ was used. In comparison, the experiments in ref 24 used YOYO-1 concentrations of $\sim 1 \mathrm{nM}$ in order to give a coverage sufficient to observe discrete single dye molecules (dye distance $\gg 1$ $\mu \mathrm{m}$, corresponding to a coverage of $<1$ dye molecule for every $\sim 3000$ base pairs). Thus, despite the increased YOYO-1 concentration used in the current work, our dye coverage is still very low (expected $\ll 1$ dye molecule for every 10 base pairs). Furthermore, we note that when the dye coverage is increased further, significant distortions in the force-extension curves are observed (see Figure S5 in the Supporting Information). Under the conditions used in our current work, we do observe distortions to the force-extension curve, but these occur at significantly greater relative extensions within the OST. Due to photo-nicking, we were unable to obtain (emission) polarization-resolved images of TC-DNA stretched along different directions within the image plane.
This limitation prevented us from quantitatively estimating probe tilt $(\theta)$ without also making assumptions about the extent of probe "wobble" $(\alpha)$. See ref 24 for a precise definition of these parameters. Nevertheless, the large magnitude, negative LD values that we obtain for TC-DNA constructs are unambiguous, in which they clearly demonstrate that intercalated dyes must align closer to the plane perpendicular to the DNA axis than was observed in the case of UC-DNA stretched to similar forces above the OST.

To further substantiate our conclusions, we performed molecular dynamics simulations of bare (unintercalated) UCDNA and TC-DNA stretched beyond the OST. These constructs were 200 base pairs in length containing AT-rich and GC-rich subregions and solvated by a continuum model (see the Supporting Information). Here, we sought to determine whether the structural motifs featuring inclined base pairs would form in either UC-DNA or TC-DNA upon stretching beyond the OST. In both of these simulated constructs, base pairing was present in GC-rich regions and exhibited inclinations of $\theta \sim 40-50^{\circ}$ (in comparison, our previous work ${ }^{24}$ determined that intercalated dyes in UC-DNA are tilted at $\theta \sim 54^{\circ}$ ). However, we caution that our simulations did not recover exact canonical forms of S-DNA and P-DNA. Specifically, base-paired regions in the simulated DNA constructs were overwound relative to the measured helicity of S-DNA ( $\sim 37.5 \mathrm{bp} / \mathrm{turn})$, and nonbase-paired regions were accordingly underwound relative to P-DNA ( $2.5 \mathrm{bp} /$ turn ). Additionally, more base-pair melting occurred (predominantly in AT-rich DNA) upon overstretching in both the simulated TC-DNA and UC-DNA than would be experimentally expected for our high-ionic-strength buffer (1 $\mathrm{M} \mathrm{NaCl}){ }^{7}$ It is not unexpected that we failed to observe purely canonical S- and P-DNA structures from simulations since the stretching forces are higher in the simulations and the construct is shorter than that in experiments. These constraints are an inevitable consequence of enabling computational tractability for atomic-level dynamic precision. Nevertheless, these simulations support our claim that S-DNA contains inclined base pairs and exists in both TC-DNA and UC-DNA. Building on these promising preliminary findings, we plan to develop further simulations in the future to include the effect of the solvent, loading rate, and intercalation.

In conclusion, we have shown that combined fluorescence polarization microscopy and DNA force spectroscopy provide a powerful means of investigating the nanoscale structural features of overstretched DNA. Our results provide a tantalizing glimpse of the different conformations that form in TC-DNA under mechanical strain and support previous predictions that these structures are highly sequence-dependent. $^{11,14,47}$ It is our hope that the new data presented here motivate further inquiry and discussion. More broadly, our current work, alongside emerging techniques in polarization microscopy, ${ }^{48,49}$ single-molecule orientation microscopy, ${ }^{50-54}$ and spatio-angular image analysis, ${ }^{55,56}$ provides a methodological blueprint to nondestructively investigate a wide variety of biological and material systems.

\section{ASSOCIATED CONTENT}

\section{(s) Supporting Information}

The Supporting Information is available free of charge at https://pubs.acs.org/doi/10.1021/acs.jpcb.1c02708. 
Simulations of TC- and UC-DNA; additional example of sequence-dependent LD in a TC-DNA molecule; additional examples of supercoiled DNA molecules; and effect of dye coverage on TC-DNA force versus extension behavior (PDF)

\section{AUTHOR INFORMATION}

\section{Corresponding Authors}

Adam S. Backer - Apple Inc, Cupertino, California 95014, United States; 이이이.org/0000-0002-3405-0984; Email: a.s.backer@gmail.com

Erwin J. G. Peterman - Department of Physics and Astronomy, LaserLaB Amsterdam, Vrije Universiteit Amsterdam, Amsterdam $1081 \mathrm{HV}$, The Netherlands; ○ orcid.org/0000-0003-1058-249X; Email: e.j.g.peterman@vu.nl

\section{Authors}

Graeme A. King - Institute of Structural and Molecular Biology, University College London, London WC1E 6BT, U.K.

Andreas S. Biebricher - Department of Physics and Astronomy, LaserLaB Amsterdam, Vrije Universiteit Amsterdam, Amsterdam $1081 \mathrm{HV}$, The Netherlands

Jack W. Shepherd - Department of Physics, University of York, York YO10 5DD, U.K.; Department of Biology, University of York, York YO10 5DD, U.K.

Agnes Noy - Department of Physics, University of York, York YO10 5DD, U.K.; (1) orcid.org/0000-0003-0673-8949

Mark C. Leake - Department of Physics, University of York, York YO10 5DD, U.K.; Department of Biology, University of

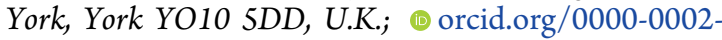
1715-1249

Iddo Heller - Department of Physics and Astronomy, LaserLaB Amsterdam, Vrije Universiteit Amsterdam, Amsterdam $1081 \mathrm{HV}$, The Netherlands; (1) orcid.org/00000003-4888-2244

Gijs J. L. Wuite - Department of Physics and Astronomy, LaserLaB Amsterdam, Vrije Universiteit Amsterdam, Amsterdam $1081 \mathrm{HV}$, The Netherlands

Complete contact information is available at:

https://pubs.acs.org/10.1021/acs.jpcb.1c02708

\section{Notes}

The authors declare the following competing financial interest(s): The combined optical tweezers fluorescence microscopy technology is licensed to LUMICKS b.v., in which I.H., G.J.L.W. and E.J.G.P. have a financial interest.

\section{ACKNOWLEDGMENTS}

Data appearing in this manuscript were collected, while A.S.Ba. was an employee of Sandia National Laboratories. A.S.Ba. acknowledges the support from the Laboratory Directed Research and Development program and the Harry S. Truman Fellowship at Sandia. This work was supported by a Chemical Sciences Top grant from the Netherlands Organization for Scientific Research (NWO) (G.J.L.W. and E.J.G.P. and G.A.K.). I.H. acknowledges research funding from an NWO VIDI award. M.C.L. acknowledges the Leverhulme Trust, U.K. (RPG-2017-340 and RPG-2019-156) and the Engineering and Physical Sciences Research Council, U.K. (EPSRC, EP/ N027639/1) (A.N. and M.C.L.), with computational time secured on JADE via the U.K. High-End Computing Consortium for Biomolecular Simulation, HECBioSim (EP/ R029407/1) (A.N.) and on the Cambridge Tier-2 system capital grant (EP/P020259/1) (A.N.). We also thank Tier 3 High-Performance Computing (HPC) facilities at the University of York, U.K. (Viking cluster) for additional computational resources.

\section{REFERENCES}

(1) Cluzel, P.; Lebrun, A.; Heller, C.; Lavery, R.; Viovy, J.-L.; Chatenay, D.; Caron, F. o. DNA: An Extensible Molecule. Science 1996, 271, 792-794.

(2) Smith, S. B.; Cui, Y.; Bustamante, C. Overstretching B-DNA: The Elastic Response of Individual Double-Stranded and SingleStranded DNA Molecules. Science 1996, 271, 795-799.

(3) Cocco, S.; Yan, J.; Léger, J.-F.; Chatenay, D.; Marko, J. F. Overstretching and force-driven strand separation of double-helix DNA. Phys. Rev. E: Stat., Nonlinear, Soft Matter Phys. 2004, 70, 011910.

(4) van Mameren, J.; Gross, P.; Farge, G.; Hooijman, P.; Modesti, M.; Falkenberg, M.; Wuite, G. J. L.; Peterman, E. J. G. Unraveling the structure of DNA during overstretching by using multicolor, singlemolecule fluorescence imaging. Proc. Natl. Acad. Sci. U.S.A. 2009, 106, $18231-18236$.

(5) Gross, P.; Laurens, N.; Oddershede, L. B.; Bockelmann, U.; Peterman, E. J. G.; Wuite, G. J. L. Quantifying how DNA stretches, melts, and changes twist under tension. Nat. Phys. 2011, 7, 731-736.

(6) Bosaeus, N.; El-Sagheer, A. H.; Brown, T.; Smith, S. B.; Åkerman, B.; Bustamante, C.; Nordén, B. Tension induces a basepaired overstretched DNA conformation. Proc. Natl. Acad. Sci. U.S.A. 2012, 109, 15179-15184.

(7) King, G. A.; Gross, P.; Bockelmann, U.; Modesti, M.; Wuite, G. J. L.; Peterman, E. J. G. Revealing the competition between peeled ssDNA, melting bubbles, and S-DNA during DNA overstretching using fluorescence microscopy. Proc. Natl. Acad. Sci. U.S.A. 2013, 110, 3859-3864.

(8) Zhang, X.; Chen, H.; Le, S.; Rouzina, I.; Doyle, P. S.; Yan, J. Revealing the competition between peeled ssDNA, melting bubbles, and S-DNA during DNA overstretching by single-molecule calorimetry. Proc. Natl. Acad. Sci. U.S.A. 2013, 110, 3865-3870.

(9) Léger, J. F.; Romano, G.; Sarkar, A.; Robert, J.; Bourdieu, L.; Chatenay, D.; Marko, J. F. Structural transitions of a twisted and stretched DNA molecule. Phys. Rev. Lett. 1999, 83, 1066-1069.

(10) Allemand, J. F.; Bensimon, D.; Lavery, R.; Croquette, V. Stretched and overwound DNA forms a Pauling-like structure with exposed bases. Proc. Natl. Acad. Sci. U.S.A. 1998, 95, 14152-14157.

(11) King, G. A.; Peterman, E. J. G.; Wuite, G. J. L. Unraveling the structural plasticity of stretched DNA under torsional constraint. Nat. Commun. 2016, 7, 11810.

(12) Vladescu, I. D.; McCauley, M. J.; Nuñez, M. E.; Rouzina, I.; Williams, M. C. Quantifying force-dependent and zero-force DNA intercalation by single-molecule stretching. Nat. Methods 2007, 4, 517-522.

(13) Biebricher, A. S.; Heller, I.; Roijmans, R. F. H.; Hoekstra, T. P.; Peterman, E. J. G.; Wuite, G. J. L. The impact of DNA intercalators on DNA and DNA-processing enzymes elucidated through forcedependent binding kinetics. Nat. Commun. 2015, 6, 7304.

(14) Schakenraad, K.; Biebricher, A. S.; Sebregts, M.; ten Bensel, B.; Peterman, E. J. G.; Wuite, G. J. L.; Heller, I.; Storm, C.; van der Schoot, P. Hyperstretching DNA. Nat. Commun. 2017, 8, 2197.

(15) Lerman, L. S. Structural considerations in the interaction of DNA and acridines. J. Mol. Biol. 1961, 3, 18-30.

(16) Glazer, A. N.; Rye, H. S. Stable dye-DNA intercalation complexes as reagents for high-sensitivity fluorescence detection. Nature 1992, 359, 859-861.

(17) Carlsson, C.; Larsson, A.; Jonsson, M.; Albinsson, B.; Nordén, B. Optical and photophysical properties of the oxazole yellow DNA probes YO and YOYO. J. Phys. Chem. 1994, 98, 10313-10321. 
(18) Larsson, A.; Carlsson, C.; Jonsson, M.; Albinsson, B. Characterization of the binding of the fluorescent dyes $\mathrm{YO}$ and YOYO to DNA by polarized light spectroscopy. J. Am. Chem. Soc. 1994, 116, 8459-8465.

(19) Bennink, M. L.; Schärer, O. D.; Kanaar, R.; Sakata-Sogawa, K.; Schins, J. M.; Kanger, J. S.; de Grooth, B. G.; Greve, J. Single-molecule manipulation of double-stranded DNA using optical tweezers: Interaction studies of DNA with RecA and YOYO-1. Cytometry, Part A 1999, 36, 200-208.

(20) Murade, C. U.; Subramaniam, V.; Otto, C.; Bennink, M. L. Force spectroscopy and fluorescence microscopy of dsDNA-YOYO-1 complexes: implications for the structure of dsDNA in the overstretching region. Nucleic Acids Res. 2010, 38, 3423-3431.

(21) Valades Cruz, C. A.; Shaban, H. A.; Kress, A.; Bertaux, N.; Monneret, S.; Mavrakis, M.; Savatier, J.; Brasselet, S. Quantitative nanoscale imaging of orientational order in biological filaments by polarized superresolution microscopy. Proc. Natl. Acad. Sci. U.S.A. 2016, 113, E820-E828.

(22) Backer, A. S.; Lee, M. Y.; Moerner, W. E. Enhanced DNA imaging using super-resolution microscopy and simultaneous singlemolecule orientation measurements. Optica 2016, 3, 659-666.

(23) van Mameren, J.; Vermeulen, K.; Wuite, G. J. L.; Peterman, E. J. G. A polarized view on DNA under tension. J. Chem. Phys. 2018, 148, 123306.

(24) Backer, A. S.; Biebricher, A. S.; King, G. A.; Wuite, G. J. L.; Heller, I.; Peterman, E. J. G. Single-molecule polarization microscopy of DNA intercalators sheds light on the structure of S-DNA. Sci. Adv. 2019, 5, No. eaav1083.

(25) Lebrun, A.; Lavery, R. Modelling extreme stretching of DNA. Nucleic Acids Res. 1996, 24, 2260-2267.

(26) Konrad, M. W.; Bolonick, J. I. Molecular dynamics simulation of DNA stretching is consistent with the tension observed for extension and strand separation and predicts a novel ladder structure. J. Am. Chem. Soc. 1996, 118, 10989-10994.

(27) Kosikov, K. M.; Gorin, A. A.; Zhurkin, V. B.; Olson, W. K. DNA stretching and compression: Large-scale simulations of double helical structures. J. Mol. Biol. 1999, 289, 1301-1326.

(28) Prévost, C.; Takahashi, M.; Lavery, R. Deforming DNA: from physics to biology. ChemPhysChem 2009, 10, 1399-1404.

(29) Danilowicz, C.; Limouse, C.; Hatch, K.; Conover, A.; Coljee, V. W.; Kleckner, N.; Prentiss, M. The structure of DNA overstretched from the 5'5' ends differs from the structure of DNA overstretched from the 3'3' ends. Proc. Natl. Acad. Sci. U.S.A. 2009, 106, 1319613201.

(30) Bosaeus, N.; Reymer, A.; Beke-Somfai, T.; Brown, T.; Takahashi, M.; Wittung-Stafshede, P.; Rocha, S.; Nordén, B. A stretched conformation of DNA with a biological role? Q. Rev. Biophys. 2017, 50, No. e11.

(31) King, G. A.; Burla, F.; Peterman, E. J. G.; Wuite, G. J. L. Supercoiling DNA Optically. Proc. Natl. Acad. Sci. U.S.A. 2019, 116, 26534-26539.

(32) Sarlós, K.; Biebricher, A. S.; Bizard, A. H.; Bakx, J. A. M.; Ferreté-Bonastre, A. G.; Modesti, M.; Paramasivam, M.; Yao, Q.; Peterman, E. J. G.; Wuite, G. J. L.; et al. Reconstitution of anaphase DNA bridge recognition and disjunction. Nat. Struct. Mol. Biol. 2018, $25,868-876$.

(33) Ha, T.; Enderle, T.; Chemla, S.; Selvin, R.; Weiss, S. Single molecule dynamics studied by polarization modulation. Phys. Rev. Lett. 1996, 77, 3979.

(34) Sosa, H.; Peterman, E. J. G.; Moerner, W. E.; Goldstein, L. S. B. $\mathrm{ADP}$-induced rocking of the kinesin motor domain revealed by singlemolecule fluorescence polarization microscopy. Nat. Struct. Mol. Biol. 2001, 8, 540-544.

(35) Peterman, E. J. G.; Sosa, H.; Goldstein, L. S. B.; Moerner, W. E. Polarized fluorescence microscopy of individual and many kinesin motors bound to axonemal microtubules. Biophys. J. 2001, 81, 28512863.

(36) Gross, P.; Farge, G.; Peterman, E. J. G.; Wuite, G. J. L. Combining optical tweezers, single-molecule fluorescence micros- copy, and microfluidics for studies of DNA-Protein interactions. Methods Enzymol. 2010, 475, 427-453.

(37) Candelli, A.; Hoekstra, T. P.; Farge, G.; Gross, P.; Peterman, E. J. G.; Wuite, G. J. L. A toolbox for generating single-stranded DNA in optical tweezers experiments. Biopolymers 2013, 99, 611-620.

(38) https://www.mathworks.com/help/signal/ref/sgolayfilt.html (accessed on March 21, 2021).

(39) Sanger, F.; Coulson, A. R.; Hong, G. F.; Hill, D. F.; Petersen, G. B. Nucleotide sequence of bacteriophage $\lambda$ DNA. J. Mol. Biol. 1982, 162, 729-773.

(40) https://www.mathworks.com/help/stats/analysis-of-varianceand-covariance.html (accessed on March 21, 2021).

(41) Axelrod, D. Carbocyanine dye orientation in red cell membrane studied by microscopic fluorescence polarization. Biophys. J. 1979, 26, $557-573$.

(42) Irving, M. Steady-state polarization from cylindrically symmetric fluorophores undergoing rapid restricted motion. Biophys. J. 1996, 70, 1830-1835.

(43) Dickson, R. M.; Norris, D. J.; Moerner, W. E. Simultaneous imaging of individual molecules aligned both parallel and perpendicular to the optic axis. Phys. Rev. Lett. 1998, 81, 5322-5325.

(44) Böhmer, M.; Enderlein, J. Orientation imaging of single molecules by wide-field epifluorescence microscopy. J. Opt. Soc. Am. B 2003, 20, 554-559.

(45) Backer, A. S.; Moerner, W. E. Extending single-molecule microscopy using optical Fourier processing. J. Phys. Chem. B 2014, $118,8313-8329$.

(46) Shroder, D. Y.; Lippert, L. G.; Goldman, Y. E. Single molecule optical measurements of orientation and rotations of biological macromolecules. Methods Appl. Fluoresc. 2016, 4, 042004.

(47) Shepherd, J. W.; Greenall, R. J.; Probert, M. I. J.; Noy, A.; Leake, M. C. The emergence of sequence-dependent structural motifs in stretched, torsionally-constrained DNA. Nucleic Acids Res. 2020, 48, $1748-1763$

(48) Brockman, J. M.; Blanchard, A. T.; Pui-Yan, V.; Derricotte, W. D.; Zhang, Y.; Fay, M. E.; Lam, W. A.; Evangelista, F. A.; Mattheyses, A. L.; Salaita, K. Mapping the 3D orientation of piconewton integrin traction forces. Nat. Methods 2018, 15, 115-118.

(49) Zhanghao, K.; Chen, X.; Liu, W.; Li, M.; Liu, Y.; Wang, Y.; Luo, S.; Wang, X.; Shan, C.; Xie, H.; et al. Super-resolution imaging of fluorescent dipoles via polarized structured illumination microscopy. Nat. Commun. 2019, 10, 4694.

(50) Mehta, S. B.; McQuilken, M.; La Rivière, P. J.; Occhipinti, P.; Verma, A.; Oldenbourg, R.; Gladfelter, A. S.; Tani, T. Dissection of molecular assembly dynamics by tracking orientation and position of single molecules in live cells. Proc. Natl. Acad. Sci. U.S.A. 2016, 113, E6352-E6361.

(51) Ding, T.; Wu, T.; Mazidi, H.; Zhang, O.; Lew, M. D. Singlemolecule orientation localization microscopy for resolving structural heterogeneities between amyloid fibrils. Optica 2020, 7, 602-607.

(52) Lu, J.; Mazidi, H.; Ding, T.; Zhang, O.; Lew, M. D. Singlemolecule orientation imaging reveals nanoscale compositional heterogeneity in lipid membranes. Angew. Chem. Int. Ed. 2020, 59, $17572-17579$.

(53) Curcio, V.; Alemán-Castañeda, L. A.; Brown, T. G.; Brasselet, S.; Alonso, M. A. Birefringent Fourier filtering for single molecule coordinate and height super-resolution imaging with dithering and orientation. Nat. Commun. 2020, 11, 5307.

(54) Hulleman, C. N.; Thorsen, R. Ø.; Stallinga, S.; Rieger, B. Simultaneous orientation and 3D localization microscopy with a vortex point spread function. bioRxiv 2020, DOI: 10.1101/ 2020.10.01.322834.

(55) Chandler, T.; Shroff, H.; Oldenbourg, R.; La Rivière, P. Spatioangular fluorescence microscopy I: basic theory. J. Opt. Soc. Am. A 2019, 36, 1334-1345.

(56) Zhang, O.; Lew, M. D. Fundamental limits on measuring the rotational constraint of single molecules using fluorescence microscopy. Phys. Rev. Lett. 2019, 122, 198301. 